\title{
GRAVITY AND THE CELLS OF GRAVITY RECEPTORS IN MAMMALS
}

\author{
M. D. Ross \\ Department of Anatomy and Cell Biology, University of Michigan, \\ Ann Arbor, MI 48109, U.S.A.
}

\section{ABSTRACT}

Two new findings, that crystals located in the inner ear gravity receptors of mammals have the internal organization requisite for the piezoelectric property, and that sensory hair cells of these same receptors possess contractile-appearing striated organelles, have prompted the author to model mammalian gravity receptors in the ear on the principles of piezoelectricity and bioenergetics. This model is presented and a brief discussion of its implications for the possible effects of weightlessness follows.

\section{INTRODUCTION}

There is only one kind of sense organ whose sole, normal stimulus would be virtualiy absent in outer space: a pure gravity receptor. The gravity receptors of vertebrates, so far as we know, do not serve this single function in any species, but respond also to dynamic linear acceleration, and, in some cases, to acoustic (vibratory) stimulation as well. Space flight will provide a unique opportuntty, then, to study gravity receptor responses to these stimuli in the virtual absence of the gravitational vector. This will yleld significant, new information virtually impossible to obtain on earth.

Aside frow the need to learn more about how a major sensory system works in the nearly complete absence of a primary stimulus, there are other compelling reasons to study gravity receptors under the auspices of the space orogram. An obvious question is whether gravity receptors, having evolved in a gravitational field to help organiams orient themselves with reference to the surface of the Earth, will degenerate through non-use under near-zero gravity conditions. This would not matter to animals living and dying in space stations, but could be of consequence to astronauts returning to the Earth's gravity after prolonged perlods of space flight. A related issue is the problem of the genesis of space-motion sickness, a syndrome to which roughly half of our astronauts appear to be susceptible. Space-motion sickness is a specific disease entity that includes perceptual disturbances as well as the nausea common to terrestrial motion sickness. Its etjology is unknown, but shifts of body fluids to the head and central mismatches in visual, kinesthetic and vestibular information have been cited [1] as likely candidates.

The role of inner ear gravity receptors in the genesis of the syndrome is often minimized and one reads (or hears) now and then [2] that the gravity receptors are shutdown in outer space. This could be argued even if the end organs were functional only in detection of the direction of gravity. However, gravity provides background stimulation against which inertial forces, generated during dynamic linear acceleration, must operate. It is a simple matter to deduce that what gravity receptors are exposed to in outer space during dynamic linear acceleration is not a resultant of two forces, but only the effect of the applied linear acceleration. The gravitational vector is lacking. Thus, it is clear that human and other vertebrate gravity receptors continue to function in outer space, but that they detect novel signals and transmit unfamiliar information to the central nervous system. It is entirely possible that, until adaptation sets in, some problems will occur in reflex coordination of movement based upon perception of the head in space. This may contribute to the generation of space-motion sickness.

But will loss of normal, vectorial signals have any permanent effect on the receptor areas themselves? We have little information on this point from experiments conducted thus far. Russian investigators first demonstrated deleterious changes in rat vestibular receptors as a consequence of space flight [3] and in later experiments found no effect [4]. The vestibular system of frogs that were fertilized and developed during space flight were also said to be normal [4].

Our own experiments may eventually provide further information on the effects of short-term space flight on gravity receptor integrity, but the effects of long-term flight are possibly more important and also are more difficult to predict. To help in the formulation of possible experiments I have begun to model the gravity receptors along piezoelectric and/or bioenergetis principles [5,6] in which different forms of energy are interchangeable. This new concept is based in part upon recent findings not yet fully published that otoconia are not single 
crystals and could be piezoelectric [7], and that striated, contractile-appearing organelles exist in the hair cells of the inner ear [8]. These new findings led me to investigate pertinent literature from other disciplines where I found much I thought to be of relevance to understanding how vestibular receptors of mamals, including those of humans, are organized and function. This paper represents a first attempt to incorporate some of the findings and ideas from these other disciplines into a model of the gravity receptor system, recognizing full well that any model so conceived will fall short of reality initially. The model will be described following a brief outline of the organization of gravity receptors in mammals. The model will then be used as the basis for a discussion of the possible effects of weightlessness on mammalian gravity receptor systems.

\section{MAMMALIAN GRAVITY RECEPTORS}

Location and general organization. Mammals have two gravity receptors on each side of the head. The receptor end organs are located in the saccule and the utricle of the vestibular membranous labyrinth of the inner ear (FIg. 1), which lies buried in the temporal bone. When viewed from above, the utricular gravity receptor end organ has the general shape of a $111 \mathrm{y}$ pad, while the saccular receptor organ is elongated but has a hook-like portion anterforly (Figs. 2a,b).

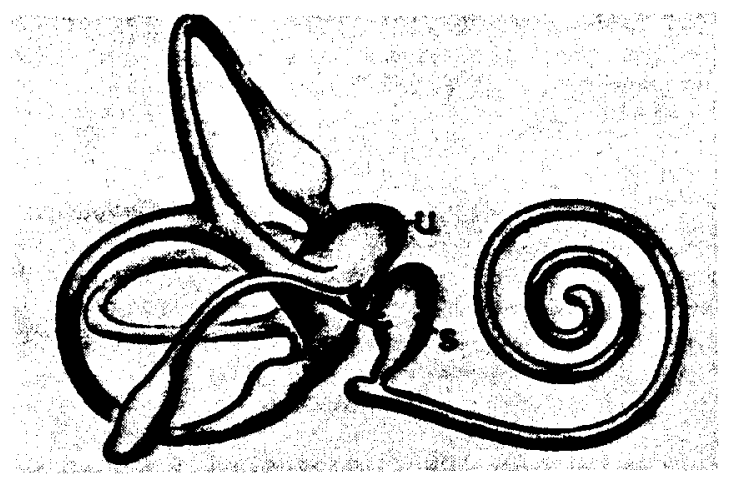

F1g. 1. The membranous labyrinth of the mamalian inner ear is diagrammed here. The gravity receptor end organs are located in the saccule (S) and the utricle (U).

Each end organ is compised of two major parts: a macula, which is a patch of neuroepithelium containing receptor hair cells and supporting cells; and a suprastructure, an otoconial complex, consisting of proteinaceous organic substance (an otoconial membrane) and a complement of minute mineral masses called otoconia. It is commonly accepted that the mineral deposits in the otoconial complexes add mass to the organic membranes, increasing the sensitivity of the underlying receptor areas to forces of gravitational and dynamic Iinear acceleration.

\section{Macular ultrastructure.}

The ultrastructure of the maculas will be described first. Each macula contains two types of hait cells (Type I and Type II) and supporting cells (Figs. 2 and 3 ).

Hair cells. Type I hair cells are somewhat flask-shaped except for their rounded lower borders while Type II hair cells are more cylindrical in outline (Fig. 3). Both kinds of hair cells have surface modifications apically from which their name as "hair" cells has been derfved. These are the ciliary tufts consisting of from 50-100 stereocilia of graded height (the number is largely species dependent) and a single kinocilium located at one side of the stereociliary bundle (F1g. 3). The stereocilia are tapered, being broader at their tips than at their bases, and are said to be very stiff in the living condition $[9,10]$. They contain highly ordered actin filaments [11] which have a liquid transverse order but are paracrystalline in the axial direction [12].

The stereocilia of the hatr cells are really long villous processes and do not resemble immotile cilia of the same name found in other regions of the body (see also Sleigh [13]). In contrast, the kinocilium corresponds in its substructure to motile cilia and flagella. It has a basal body and striated rootlets extending into the cytoplasm below [8]. Motility of the hair cell kinocilium has been observed under experimental conditions [10] but whether it is ordinarily motile and plays a functional role in hair cell transduction is not known with certalnty (see below, HAIR CELL TRANSDUCTION).

The stereocilia have electron dense rootlets consisting of packed actiñ filaments [11] that project into the cuticular plate in the apical region of the cell. The cuticular plate, according to our recent observations $[8]$, is a striated organelle that lies under the stereociliary tuft. The basal body of the kinociliary complex lies in a cuticular-free zone of cytoplasm, although it has filamentous attachments to small aggregates of electron dense, 

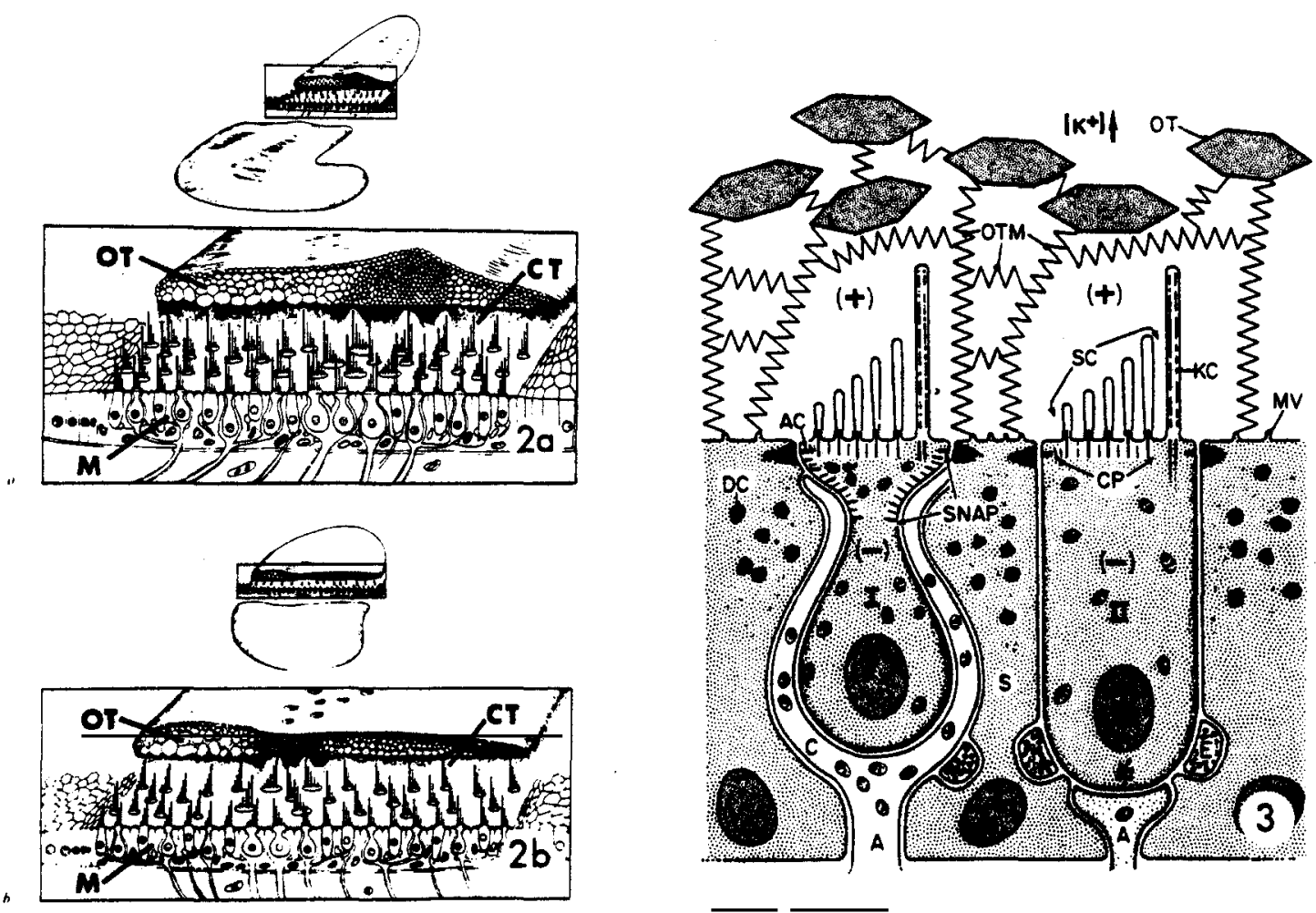

Figs. 2a,b. These diagrams schematically show the arrangement of the otoconia (OT) and the macular cells ( $M$ ) of the saccular (a) and the utricular (b) gravity receptors. CT, cillary tuft. (With permission of H.H.Lindemann and Springer-Verlas [37].)

Fig. 3. The ultrastructural organization of a mammalian gravity receptor end organ is illustrated here. The otoconial membrane (OTM) is depicted as a number of springs that are attached to the microvilli (MV) of the supporting cells (S) of the macula below and to the otoconia (OT) above. The macula consists of Type I (I) and Type II (II) hair cells and supporting cells (S). The fluid around the otoconia (endolymph) has a high concentration of potassium ions $\left(\left[\mathrm{K}^{+}\right] \mathrm{h}\right.$ ) but is low in sodium ions. A standing potential exists between endolymph $(+)$ and the interior of the hatr cells $(-)$. See text for further details. A, afferent nerve ending; $A C$, actin condensation internal to zonula adherens; $C$, calyx nerve ending; $C P$, cuticular plate; DC, dilated cisternae; $E$, efferent nerve ending; KC, kinocilium; SC, stereocilla; SNAP, striated neck apparatus. The lines in $C P$ and in SNAP indicate only that striations are present but are not meant to depict details of the direction of the striations.

material at the border of the cuticular plate [8]. Basally, the cuticular plate is connected to condensations of actin fllaments [14] that lie just internal to the zonula adherens.

Dynamic polarization of hair cells. The position of the kinocillum and its basal body is said to determine the dynamic polarization of the cell. That 18 , bending the stereocilia in the direction of the kinocilium has been shown experimentally to depolarize the hair cell and increase its activity while bending of the stereocilia in the opposite direction hyperpolarizes the cell and decreases its activity $[15,16,17]$. The kinocilla point in opposite directions in each half of a macula, with the division occurring along a curved line called a striola (Figs. $4 a, b)[9,16,18]$.

Differences between Iype I and Type II hair cells. There are several differences between the two kinds of hair cells in internal organization [19] and in the way nerve fibers relate to them. Only those differences pertinent to the present discussion will be taken up here. Microtubules emanate from the cuticular plate and extend distally in the Type I hair cells (Fig. 5) so that the cuticular plate likely corresponds to a microtubule organizing region [8]. This would explain Zenner's [20] finding of tubulin in the cuticular plate, especially in the Type I hair cell. In contrast, Type II hair cells have more prominent arrays of smooth endoplasmic reticulum and fewer microtubules apically (Fig. 6).

We have shown recently that Type I hair cells of the adult rat utricular model contain an elaborate, striated organelle that extends far into the neck region of the cell [8] (Fig.5). We have named this organelle "striated neck apparatus" (SNAP). This apparatus lies apposed 



Figs. 4a and $b$ fllustrate the direction of polarization of the hair cells of the utricle (a) and saccule (b). The central dotted line defines the striola, along which direction of polarization changes (From "Ultrastructure of the Vestibular Sense Organ", by H. Spoendlin in THE VESTIBULAR SYSTEM AND ITS DISEASES, ed. Robert J. Wolfson, University of Pennsylvania Press, Philadelphia, 1966)


Fig. 5. This electron micrograph shows a portion of the striated organelle of Type I hair cells (arrow). In this oblique section, the striated appearance of the cuticular plate (CP) is not clear. Note the abundant mitochondria (M) and microtubules (MT) in the fleld. C, calyx nerve ending. The bar equals $1 \mu \mathrm{m}$.

Fig. 6. A portion of the apical region of a Type II hair cell is illustrated here. Note the general orientations of the electron dense material parallel to the cell surface (see espectally area between pointers). Connections of the cuticular plate (CP) to the actin condensation (AC) at the border of the cell are also evident (arrow heads). Note the small mitochondria (M) and widely spaced microtubules (MT). Compare with F1g. 5. The bar equals $1 \mu \mathrm{m}$.

to the plasma membrane, to which it is probably cross-linked, directly under the upper end of the afferent nerve ending (the calyx, see below). While the three-dimensional organization of the strfated neck apparatus has not been determined, it seems that Type II hair cells lack an organelle of comparable magnitude (Fig. 6).

A further difference we have noted in the case of the rat utricular hair cells is the presence of minute bodies, resembling miniature otoconia, in the cuticular-free zone of the cell near the kinocilium in some Type II halr cells. These masses may be "cores" around which otoconial formation could take place once the bodies are discharged into the area above the macula.

Innervation. Wuch of the Type I hair cell is surrounded by a cup-shaped, sensory (afferent) nerve terminal that is called a calyx because of its resemblance to the calyx of a flower (Fig. 3). Type II hair cells have, instead, small, button-like nerve terminals (boutons terminaux) ending upon them. Some of these are afferent but others contain vesicles and are of efferent (effector) type (Fig. 3). Efferent-type nerve terminals end upon the calyces also but do not terminate directly on Type I halr cells (Fig. 3). The afferent terminals 
are branches of the statoacoustic nerve, while the origin of the efferent-type terminals is disputed (central nervous system [21]; vestibular ganglion [22]).

Supporting cells. The supporting cells intervene between the halr cells. Their functions are largely unknown. The apical regions of these cells contain many dilated cisternae filled with a granular substance (Fig. 3). Thus, these cells may contribute organic substance to the otoconial membrane.

\section{Otoconial complex ultrastructure}

The otoconial membrane and the otoconia together constitute an otoconial complex. The otoconial membrane consists of proteinaceous organic material that serves to attach the otoconial mass to the supporting cells of the macula, but its connections to the ciliary tufts are disputed (see Lindemann [23] for discussion). In the striolar regions, at least, the ciliary tufts are not connected to the otoconial membrane but are surrounded by a fluid compartment thought to contain endolymph(see below) or a related liquid material (Figs. 2 \& 3 ). The organic substance of the otoconial membrane is continuous with the organic phase of the otoconial crystals, which consist of calcite and organic material, and with further organic matter that interconnects the otoconia (Fig. 7). The otoconia vary between 3 and $20 \mu m$ in length and are distributed by size over each macula (Fig. 3 for guinea pig). Each otoconium has the configuration of a single crystal of calcite (Fig. 7). Typically, the otoconial body is rounded or irregularly faceted, but the end faces are three in number, planar, and those of a rhombohedron [24]. Less typical are crossed (Fig. 7) or conical configurations.
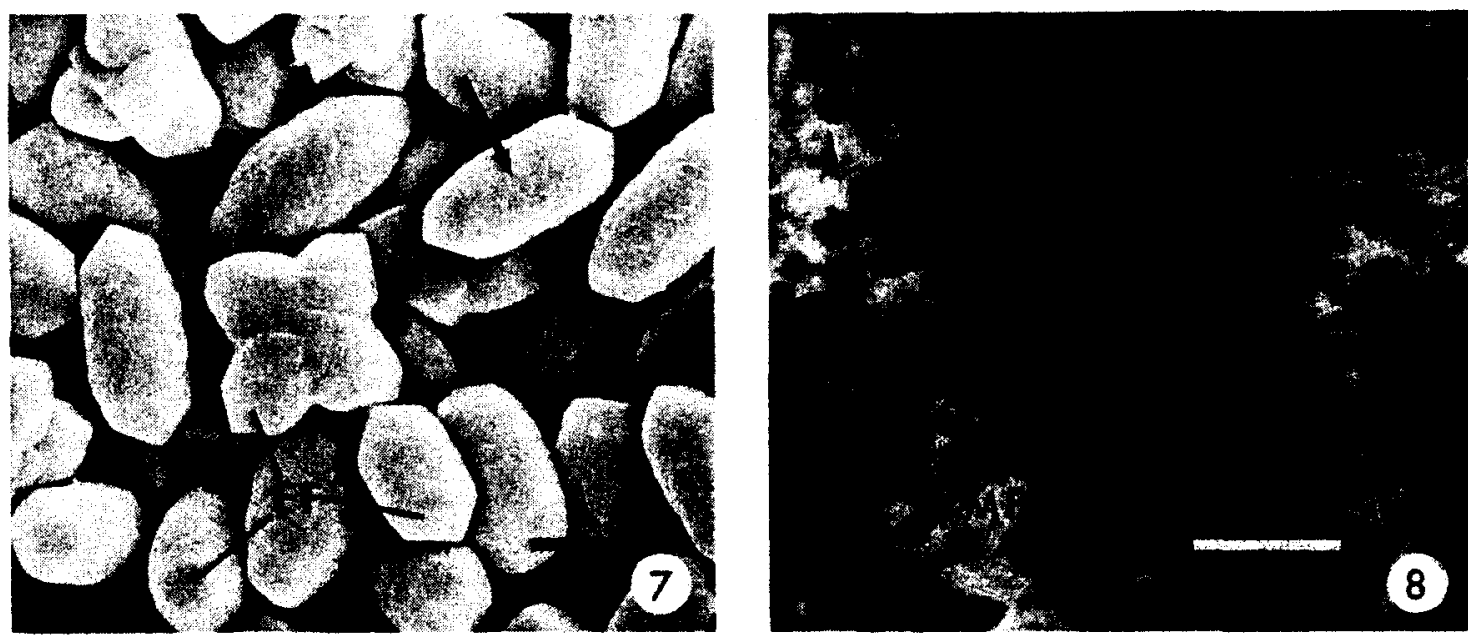

Fig. 7. This scanning electron micrograph illustrates the appearance of rat otoconia. Typically, the individual otoconia have rounded bodies (arrow) and planar, rhombohedron end faces (EF). An otoconium in the crossed configuration is present in the center of the field. The bar equals $5 \mu \mathrm{m}$.

Fig. 8. This is a transmission electron micrograph obtained from an obliquely sectioned undecalcified, unstained saccular otoconium. The crystallites of calcite are the only electron dense structures in the micrograph; the organic material between them is electron lucent because it was not stained. The crystallites have some borders that are rounded (arrow head) and are arranged in layers (see region Indicated by arrows), although this order is more clear in ultrathin fragments. The electron dense, periodic lines at upper right and at lower left, center are Moire fringes (MF). These are commonly seen on micrographs of layer lattice minerals where the angle between two overlapping crystals is small. The bar equals $660 \AA$.

\section{PIEZOELECTRIC CHARACTER OF OTOCONIA}

Otoconia have been described as behaving like single crystals on crystallographic grounds[25], largely on the basis of $x$-ray powder diffraction studies. Their morphology and chemistry prompted us to consider otoconia to be single crystals $[24,26]$, but Nakahara and Bevelander [27] thought them to be polycrystalline. Recent high resolution microscopy of fish, frog and rat inner ear minerals has demonstrated, however, that all of these mineral deposits consist of ordered laminated arrays or domains of microcrystallites [7]. The domains are slightly out of phase (translationally displaced) from one layer to the next (Fig. 8). Otoconia do not, therefore, correspond either to single crystals, in which extensive three-dimensional periodicity occurs, or to polycrystalline masses in which randomness is implicit. This new observation means that otoconia and otoliths like bone [28] and other organic mineralized tissues are possibly piezoelectric [7]. Fish otoliths were previously described as piezo- 
electric by Morris and Kittleman [29] on the basis of direct, experimental analysis.

\section{STANDING ELECTRIC POTENTIAL}

The entire membranous labyrinth is filled with a fluid called endolymph that is high in potassium tons and low in sodium ions [30]. Outside the membranous labyrinth is another fluid, perflymph, that has approximately the reverse ionic composition to endolymph (it is high in sodium and low in potassfum). When the electric potential of the endolymph is measured with respect to perilymph, it is found to be positive. There is, moreover, a considerable standing potential between the endolymph and the hair cell interior (note positive and negattve signs in Fig. 3). The standing potential has not been measured across gravity receptors to my knowledge, but it amounts to about $120 \mathrm{mV}$ in the cristae ampullares, [31] vestibular zeceptor areas that detect angular acceleration. In the cochlea, a detector of acoustic stimuli, there is a standing potential [32] of at least $120 \mathrm{mV}$ [33]. The origins of these potentials are not completely known, but a standing potential appears to be essential to inner ear hafr cell activity (see [34] for further discussion).

\section{CURRENT THEORY OF TRANSDUCTION}

According to the currently accepted theory of transduction in the gravity receptor system, static gravitational force displaces the otoconia en masse in one direction or another, depending upon the position of the head. This provides a shearing force to the stereocilia of the halr cells, perhaps bending them at their bases [17]. The direction of bending determines whether the bair cell will be activated or inhibited. Dynamic acceleration utilizes inertial displacement of the otoconial mass, which then lags behind the macula as it moves with the head. Again, a shearing force and stereociliary bending are invoked as the mechanisms underlying slgnal detection.

The kinocilium is generally discounted as playing a major role fn the transduction process [35]. However, Flock [36] has suggested that it is too early to dismiss the kinocilium as unimportant in hair cell activity.

Basically, the accepted theory holds that the mechanical energy entering the system mechanically displaces the otoconia, mechanically bending the stereocilia. Only at the level of the hair cell does transduction to electrochemical energy occur. Hudspeth [35] has proposed that initial transduction of mechanical into electrical energy occurs at the tips of the stereocilia, based upon his new finding that, in vitro, there is a decrement in extracellular potential from the top of the stereocilium down along the upper part of its shaft, when the stereocilium is mechanically stimulated.

Although the currently accepted theory deals wh the otoconia as displaced en masse, this might not be true, as Lindemann [23] has also suggested. If the otoconia are considered to be interconnected by springs of equal stiffness, and as lying in a single layer but in a distribution by size as Lindemann [37] has shown for the guinea pig, it becomes readily apparent that acceleration of the head to the right would yield different kinds of relative motions of the crystals over a given macula than would acceleration of the head to the left (F1g. 9). This is because although the acceleration is applied uniformly and the resistance to movement of the otoconia offered by the springs (or impedance) is assumed to be similar, the masses acted upon are different. The larger masses will have a greater inertia to displacement than will the smaller masses. In some cases, then, the relative motions of the masses $\mathbf{1 1 1}$ be to approximate one another and in other cases to move farther apart (Fig. 9). Addition of other springs underneath the otoconia to model the membrane stiffness imposes further restrictions on otoconial movement in any given direction. The models given here for the guinea pig macula would not be directly appllcable to all other species, because the otoconia are not distributed similarly in all cases. However, the principles would apply.

A.romomamamamamarman

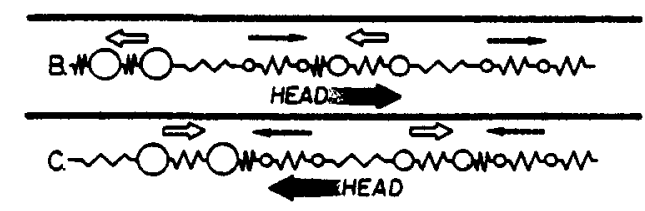

Figs. 9 A,B.C. This figure schematically shows otoconia of different sizes distributed across the utricular macula, interconnected by springs of equal stiffness. A represents the equilibrium position; in $B$, the head is moving to the right; in $C$, the head is moving to the left. The arrows above the masses indicate the relative motions of the otoconial masses. 
A NEW MODEL

Even if the gravity receptor were considered to be a pure mechanoreceptor, differences in relative motions of the otoconia could be of importance in signalling the direction of a given movement, while the rate of change in relative positions could aid the detection of the rate of acceleration. However, our two new observations, 1) that the otoconia are not single crystals and could be piezoelectric [7], and 2), that contractile-appearing, striated organelles occur in the hair cells [8] may indicate that the concept of transduction in gravity receptors currently in vogue is too mechanistic.

The present model considers that mechanical energy is first converted into electrical energy at the level of the otoconial complexes to modulate the standing potential. Changes in the electrical field then modulate hair cell activity, with kinociliary-stereociliary interactions underlying hair cell activation and transduction. Mechanical, electrical and chemical energies are largely interchangeable, which means that feedback is built into the system.

In order to explore these concepts further, and to consider the effects of near-zero gravity upon a system such as is proposed here, we must first define the piezoelectric property and principles of energy transfer as considered under the umbrella of "bioenergetics" $[5,6]$. According to Cady [38], "A piezoelectric crystal may be defined as a crystal in wh1ch 'electricity or electric polarity' is produced by pressure...or as one that becomes deformed in an electric field." The first definition expresses the direct effect; the second, the converse effect. Further, the direct piezoelectric effect is "an electric polarization produced by mechanical strain in crystals belonging to certain classes, the polarization being proportional to the strain and changing sign with it." In the converse effect, whereby a crystal becomes deformed when electrically polarized, the amount of deformation is directly proportional to the electric, polarizing field and reversal of sign takes place with reversal of field. These definitions imply that crystals exhibiting piezoelectricity must lack a center of symetry. In naturally occurring crystals, only 20 of the 32 crystallographic classes have such asymmetry to permit polarization under stress in an electric field. Naturally occurring calcite and apatite are not among those showing piezoelectricity; they have centers of symetry that extend even into their molecular, unit-cell structure. However, bone, which contains hydroxyapatite, is known to be piezoelectric [28], as are the dentine and cementum of teeth [39]. It is currently believed that it is the organic material (specifically, collagen) of bones and teeth that confers the property of piezoelectricity on them, although stress at the organic-inorganic material interfaces is another possibility [40]. Otoconia also contain organic, proteinaceous material which appears to be chemically dissimilar to collagen [41] but is or contains glycoprotein based upon its strong periodic acid Schiff (PAS) reactivity $[42,43]$.

Proteins can be purified and, in the dry state, often have three-dimensional configurations that appear crystalline. Indeed, purified proteins have of ten been studied by crystallographic methods. However, in the living organ1sm proteins are in constant fluctuation, even periodic oscillation [44]. According to Green [44], they fold and unfold, essentially producing "domains", with periodic exposure of exchangeable hydrogen ( $\mathrm{H}^{+}$) atoms situated on amino acids. They have been likened to three-dimensional springs [44] which can store mechanical or electrical energy (they act like capacitors).

Green [44] has proposed that proteins are unique, macromolecular devices for energy manipulation in biological systems. Included under the umbrella of a broadly based discipline called bioenergetics $[5,6]$ is the principle that thermal energy can be converted (transduced) by proteins into electrical, mechanical and chemical energy. Radiative energy, such as light or sound, could be converted similarly, making the bioenergetic concepts applicable to the visual and auditory systems.

\section{OTOCONIAL COMPLEX TRANSDUCTION}

Otoconial complexes have proteinaceous matter within the crystals as well as in the otoconial membrane. The protein of the membrane has already been treated here as a number of interconnected springs which could store energy but, in keeping with Green's hypothesis, could also act as a transducer. The otoconial crystals possess many lattice defects (F1g. 8) due to their protein complement which could contribute to the production of charges occurring at the interfaces of domains, or where there are "holes" or imperfections in the crystal lattices [45]. This would be in addition to surface charge induced by crystal deformation under stress and the otoconia may fall into the class of materials called semiconductors.

Also, if otoconia are piezoelectric, they do not have to be moved physically in order to transmit information concerning the direction of static gravitational force or of the direction and rate of dynamic linear acceleration. They would exist in a "set point", or state of some stress, at equilibrium conditions due to the standing potential (see p. 6) existing between the environment $(+)$ and the hair cells $(-)$, and to the stresses imposed by their attachments, both interotoconial and to the maculas. The organic material forming the largely proteinaceous attachments that act like three-dimensional springs would also be 
strained by the masses (otoconia). Adding imposed stresses through static gravitational force or dynamic linear acceleration would produce altered charge distributions in the otocontal complexes, perhaps compounded by the forced movement of sol through the gel-like otoconial membrane. This would make the electric field above the hair cells more, or less, positive at any given moment, or reverse the sign, and effect a change in the relative charge (negative [10]) on the cilia of the hair cells. The net result would be amplification of mechanical (or radiative) energy through transduction into electrical form.

To this basic concept must be added a directional component. In order for an electric field or for mechanical deformation to cause the piezoelectric effect, it usually must be directed properly; i.e., it must be directed parallel to, perpendicular to, or cangential to the crystal. According to Cady [38], there is no class of crystal in which piezoelectric polarization has only one direction, but there are several classes in which polarization is confined to a particular plane. In one class of asymetric crystals (triclinic) a random stress of any kind will produce a polarization. In the context of this discussion it would seem important to note that the otoconia are not all oriented similarly. While at first glance it might seem that any induced piezoelectric effects would be cancelled out in part because of this arrangement, it is equally possible that the randomness of orientation of the otoconia is only apparent and that in fact a closer analysis may show that there is order in the orientation. For example, particular groups of crystals might be situated to better detect specific directions of applied force. In turn, the induced changes in charge distribution (modulated electric field) would have to be directed properly toward the cilia of the hair cells to be effective maximally there.

Much experimental research will be required to determine whether otoconia and their associated proteins are piezoelectric. The problem will be compounded by the small volume of material to be tested and by the fact that the piezoelectric property is probably enhanced in the wet state, and under ionic conditfons existing in vivo, which may be difficult to reproduce exactly under experimental conditions. For example, the conductivity of crystalline hemoglobin increases by a factor of 1010 upon adsorption of $8 \%$ (by weight) of water [46]. Whether the effects to be taken up here on a theoretical basis should be called strictly piezoelectrlc or would fall more correctly under the discipline of bioenergetics can only be explored as more experimental evidence is accrued.

\section{HAIR CELL TRANSDUCTION}

It has been hypothesized here that the initial transduction of gravitational or applied dynamic linear acceleratory force occurs at the level of the otoconial complex, where mechanical energy is transduced into electrical energy to modulate the standing potential gradient in the system. The present model further considers that hair cell transduction involves modulation of kinocillary beat by the milleu, including the standing potential, and that kdnociliary-stereociliary interactions underlie signal detection and transduction in vivo in the gravity receptor system.

In contrast to the concept presented here, that the kinocillary complex is important in the hair cell transduction process, Hudspeth [35] has Indicated that neither the kinociliary shaft, its necklace, nor its basal body are essential to hair cell activation in the case of the bullfrog saccular macula. Depolarization can be induced in vitro by bending the stereocilia in the direction of the kinocilium even if the kinocilium and its basal body have been removed. Such mechanically induced hair cell depolarization does not prove or disprove the importance of kinociliary activity in coordinating bair cell electromechanochemical responses for signal detection in the living animal. This is especially true since Hudspeth's experiments were conducted under extremely artificial conditions in which a high $\mathrm{Na}^{+}$rather than high $\mathrm{K}^{+}$medium was used. It is well knowri that the lonic condittons around cilia can determine whether they beat at all, or whether they beat in normal or in reverse direction, and can influence the strength of the beat (see Sleigh [13]). $\mathrm{Mg}^{++}, \mathrm{Ca}^{++}$and $\mathrm{K}^{+}$ions are of particular importance in this regard, but especially $\mathrm{Ca}^{++}$. Critical balances of these ions are also requisite to flagellar contraction [47], to which kinocilia are analogous; and to other contractile elements such as muscle fibers and reconstituted actomyosin threads [48], to which the contractile elements we have described may be related.

Flock et al. [10] have reported that kinocillary movement ts whip-like, a type of motion that is consistent with motile clila (Sleigh [13] for discussion), and have noted (importantly) that the stereocilia followed the motion of the kinocilia. If kinocilia are motile, and there is no cogent reason to belleve otherwise based upon the ultrastructural and experimental evidence, then the milieu around them is doubtless tailored to their activity. Even if the kinocilia are surrounded by viscous material, or if kinocilia and stereocilia are interrelated by viscous macromolecules, this would not automatically preclude ciliary beating. The effects of a viscous medium on cillary beating is discussed by sleigh [13]. The evidence available from invertebrates is that a viscous medium can actually assist metachronal beating (beating in sequence of motile elements) when the viscosity is between 3.6 and $10-15 \mathrm{cP}$ (water has a viscosity of $1.002 \mathrm{cP}$ at $20^{\circ} \mathrm{C}$ ). In the case of hair cells, viscous material between the kino- and stereocilia could be used in somewhat analogous 
fashion, to influence the velocity of transfer of signals between them.

Of particular relevance to the present model is the experimental finding that in the case of motile cilia and flagella of invertebrates, ciliary (flagellar) beat can be influenced by changes in the electric field $[49,50]$. It was suggested that a uniceliular organism may act as a "core conductor" with the applied electric current affecting the membrane potential [51,52]. Later, Bovee and Jahn [53] summarized the evidence to support the concept that flagellar structures might be piezoelectric.

Stereocilia of hair cells have not been shown to deform in an applied electric field, but neither has the appropriate experiment been conducted. The interesting possibility exists that the stereocilia and kinocilia are piezoelectric or bioenergetic elements functioning in parallel and influencing one another's activities, much as Bovee and Jahn [53] have proposed for the stigma (a photosensitive organelle) and the flagellum in Euglena.

The actin of the stereocilia is probably cross-linked to the surface membrane, as in intestinal microvilli [54] (to which stereocilia bear some resemblance), where interactions with the environment could occur. It would be premature at the present time to consider that the actin filaments are always in the state of paracrystalline assembly, or "rigor", that we see in our electron microscopes or under in vitro conditions, in which the preparative procedures can govern the actin state. Much recent evidence is accruing to indicate that actin filaments are under protein regulation to form them either into bundles or into isotropic gels (see [55,56] for review). Mechanisms modulating these proteins are only now coming under intensive study. One of the proteins that stabilizes the core microfilaments in intestinal microvilli is villin [56]. In the absence of $\mathrm{Ca}^{++}\left(<10^{-7} \mathrm{M}\right)$ it crosslinks actin into bundles but when the calcium ion concentration is raised the bundles are dispersed and the filaments are fragmented. Thus, calcium ion "transients" would appear to be of great importance to actin assembly and disassembly, as they are to the production of contractile force. It is clear that, as research on actin continues in other systems, much that is new will be applicable to the study of stereocilia.

Striated organelles of hair cells. But stereocilia are not the only actin-containing elements of hair cells. Actin is present in the striated rootlets of the basal bodies, in the cutiailar plates of both kinds of hair cells and in the striated neck apparatus of the Type $I$ hatr cell. At all these sites, the actin is organized in a way in keeping with the property of contract1lity. Moreover, the actin must be in association with proteins different from those in the stereocilia, because striations are evident and also because the registered order of the actin has been more difficult to preserve by ordinary fixation methods. In contrast, the order in the stereocillary actin filaments has been known for some time.

The organization of the striated cuticular plate has not been completely worked out. It appears, however, that a portion of it has electron dense "stripes" ordered parallel to the plane of the apical surface membrane of the cell and actin microfilaments running in a direction parallel to the rootlets of the stereocilia. The rootlets are linked to the electron dense material by small bundles of filaments [8]. Thus, while stereociliary deformation would be expected to affect the actin in the cuticular plate, the converse would also be true: contraction (or tension) in the cuticular plate could affect stereociliary actin, perhaps causing fragmentation and metachronal stereociliary bending. Moreover, contraction of the cuticular plate could influence distal regions of the cells through the microtubular arrays in Type I hair cells and smooth endoplasmic reticulum in Type II hair cells.

The kinociliary apparatus as a pacemaker. The physiological route for activation of the striated organelles of the hair cells will have to be determined through future research. The effects of this activation will be of paramount importance in terms of hair cell transduction. But what seems to be essential in the hair cell system is some mechanism for pacing the cell's activities and for coordinating the interrelated elements to provide directionality. The one element of the hair cell that has the necessary machinery to accomplish this is the kinociliary complex. Evidence has accrued from the study of invertebrate motile cilia and flagella that the rate, strength and direction of ciliary beating can be influenced by electrical, mechanical and chemical factors in the environment. It is also significant that kinocilia have refractory periods during which time internal reorganization is taking place and another beat cannot be induced. Thus, the kinociliary complex could provide not only the basis for metachronal coordination of the many elements integrated into yielding the hair cell's response, but could also serve as the ultimate source of "spontaneous" firing of nerve fibers ending on the hair cells. In this regard it is of interest that ciliary beating is under neuronal control in some metazoans [13], and there are efferent-type nerve terminals on Type II hair cells and on the afferent calyces on Type I cells.

\section{MODULATION OF INNER EAR STANDING POTENTIALS AND NEURAL RESPONSES} IN THE LIVING SYSTEM

There is ample evidence in the literature that the standing potential, its modulation during natural stimulation or by imposed galvanic current, and the directional polarization of the 
potential with reference to the dynamic polarization of the hair cells are all related to the nature of the neural responses (excitation or suppression) elicited upon mechanical stimulation of the vestibular macular and ampullary end organs $[31,57,58,59]$. Indeed, the consistency of the interrelationships under various experimental conditions has led wilson and Melvill Jones [34] to suggest that some degree of causality must exist between them. A polarized direct current can also affect cochlear activity $[60,61,62,63]$.

The applicability of the present model to the other receptor organs of the inner ear will not be considered in detail here because the main focus of this article is on the gravity receptors. But it is clear that a unified theory of transduction in all these receptors is posstble on the basis of the present concepts: that conversion of mechanical or radiative energy into electrical energy is an early event and that mechanical, electrical and chemical energies are largely interchangeable in the system.

\section{CONCLUS IONS}

Whether the gravity receptors eventually are proved to function purely as mechanoreceptors or through modulation of the electric field by the otoconial complexes as is proposed here, the precise effects of near-zero gravity conditions on them is equally complicated and difficult to predict. This is partly because many questions remain about the spectfic functioning of various parts of the system. Additionally, our recent finding of the striated neck apparatus, an organelle not observed before, leads to the possibility that other elements important to hair cell activity may have escaped attention. The only real answer to the quandary is to carry out ground-based and space flight experiments and to test the observations for degree of fit to the proposed model. The model can be modified as required to taflor it to the facts as they come in.

The new model includes the concept that the otoconia not only add mass but add it in such a way that the mineral-organic complexes produced are piezoelectric and modulate the standing potential existing in the system. It differs significantly from one suggested earlfer by Offutt [64], who did however postulate the existence of a piezoelectric material in hair cells. The model predicts that the standing potential actually acting on the hair cells would be different in a space environment than in Earth's gravitational field, due to loss of the strain on the otoconial complexes ordinarily imposed by gravitational force. This can be tested. It is this "set point" or bias against which inertia acts within the setting of Earth's gravitational field and $i t$ is this bias that would be lacking in space. This would make even the perception of inertial forces during dynamic linear acceleration faulty in a space environment and could confound central integrators of vestibular signals, producing sensory conflict and some aspects of space-motion sickness.

In either the resting state or during dynamic acceleration of the head, the rate and/or strength of kinociliary beat, the configuration of the actin in stereocilia, the degree of contractility or tension in the cuticular plate and in the striated neck apparatus, and the spontaneous firing rate of. the afferent nerve fibers could all be affected by changes in the electrical field in outer space according to our model. Presently, we know too little about the interactions of all of these elements on earth to safely predict the consequence of nearzero gravity upon them. There is preclous little in the literature to provide enlightenment. To date, the only physiological experiment conducted on gravity receptors over an extended period of time (6 days) in space is one carried out by Bracchi et al. [65] on the bullfrog. Four afferent nerve fibers, identifled as tonic statoreceptors but of undetermined macular origin, were followed electrophysiologically for several hours preflight and for six and a half days in flight. Early effects (first 90 mins) of exposure to near-zero gravity were not recorded, but after that the nerve fibers showed fluctuations in resting activity and in their characteristic responses to linear acceleration. Units that were rapidly adapting, or phasic, on Earth became slowly adapting, or tonic, in orbit, and vice versa. The changes were attributed to unloading of the maculas, which were then said to respond rather freely to random forces acting upon them. The results of these experiments are too few, and details of macular origin too sketchy, to interpret them in light of our new model.

A very significant effect of the absence of gravity might be to reduce the static charge normally present on the otoconial complexes when under stress in a gravitational field, interfering with their ability to adsorb calcium ions. Over prolonged exposure to near-zero gravity conditions calcium regulation in the system might be perturbed. Calcium ton concentrations are important to any living system, but in the inner ear calcium ion transients could be critical to the modulation of kinociliary beat and of stereocillary actin configuration. Otoconial complexes take up calcium ions both in vivo and in vitro, but the uptake under in vitro conditions is very much greater [66]. These results have been difficult to interpret, but the stirring used in the in vitro experiments might have induced strains that led to increased electronegative surface charge and more adsorption of $\mathrm{Ca}^{+}$.

These and other studies show that the role of the otoconial complexes in regulating calcium ions in the membranous labyrinth may be greatly underestimated at the present time. Moreover, what occurs in the way of ion regulation by the complexes in mammals may reflect only a 
portion of that potential as expressed during evolutionary history by other vertebrates. For example, it is well known that certain anuran frogs utilize the calcium stored in otoconia located in large, endolymphatic sacs for skeletal mineralization during metamorphosis from the tadpole to the adult stage [67]. Very recently, Rask-Andersen and a colleague have provided evidence that otoconia-like bodies are present in a mammalian (guinea pig) sac during the fetal stage. Some of the otoconia-like bodies were free in the sac but others were engulfed in intrasaccular cells where they appeared to be undergoing degradation [68]. This interesting set of observations and our own, that otoconia-1ike bodies occur in the hair cells of the adult rat, raise questions relevant to otoconial turnover and utilization that space-related research may help us to answer.

Another effect of weightlessness could be changes in otoconial morphology if otoconia, like bones and teeth, are pyroelectric as well as piezoelectric. Pyroelectricity is a property of certain crystal classes whereby positive and negative polarization charges change with temperature (direct effect) or the crystals change temperature in an electric field (converse effect). The converse effect is negligible [38].

Pyroelectriclty is expected to occur in otoconial complexes because they function in a liquid milieu and only pyroelectric materials show the piezoelectric effect under hydrostatic pressure. This would mean in turn that otoconia are permanently polar1zed [69] as are bones and teeth and many other soft tissues [70]. Such permanent polarization would help explain many aspects of otoconial morphogenesis now unclear to us, such as how otoconia achieve such perfect planar end faces, and why some otoconia have crossed configurations (F18. 7) or conical shapes. In the case of bones and teeth, it is believed that growth occurs along the direction of polarization which may, however, change as maturation takes place [70].

Finaliy, we have only begun to study the striated organelles recently described [8] as present in the cuticular plates of both kinds of hair cells and in the neck region of Type I hair cells. It will be interesting to follow their morphology in animals exposed to weightlessness. That will require learning how to preserve these interesting organelies in $a$ condition to truly reflect conditions at the time of sacrifice. We do not yet know how to accomplish this. But then, it was only a short time ago that we did not know that the striated organelles existed at all.

\section{ACKNOWLEDGEMENTS}

I thank Mrs. Carol Bourne for her technical assistance, and Mr. William Brudon for Figs. 3 and 9 . This research was supported by NASA Grant NSG 9047 and NASA Contract NAS2-10535. Research on high resolution electron microscopy of otoconia was supported by a Fogarty Senior International Fellowship, NIH I F06-TW00589.

\section{REFERENCES}

1. C.M. Oman, MIT Man Vehicle Laboratory Report MVT-80-1 (1982)

2. E.V.Lapayev and G.I.Pavlov. Cited in USSR Space Life Sciences Digest, 2, 37 (1981)

3. Ya A.Vinnikov, O.G.Gazenko, L.K. Titova, A.A.Bronstein, V.I.Govardovski1, F.G.Gribakin, R.A.Pevzner, M.Z.Aronova, T.A.Kharkeevich, T.P.Tsirulis, G.G.Pyatkina, D.V.Kichakov, L.P.Pal'mbach and V.F.Arichin. Acta Otolaryngol. 87, 90 (1979)

4. Ya A.Vinnikov, D.V.Lychakov, L. R. Pal'mbakh, V.I.Govardovskiy, V. O.Adanina, B.L. Allakhverdov and A.G.Pogroelov, J. Evol. Biochem.Physiol. (Leningrad) 16, 574 (1980)

5. D.E.Green and S.Ji, Bioenergetics 3, 159 (1972)

6. D.E.Green and S.Ji, in: The Molecular Basis of Electron Transport, Academic Press, New York, 1972, p. 1

7. M.D.Ross, S.Mann, A.J.Skarnulis and R.J.P.Williams, Association for Research in Otolaryngology, St. Petersburg, Florida, January 18-21, 1982 (Abstract)

8. M.D.Ross and C.E.Bourne, Scfence (in press)

9. H. Engstrym, H.W.Ades and J.E.Hawkins, Jr., J. Acoust. Soc. Am. 34, 1356 (1962)

10. A. Flock, B.Flock and E.Murray, Acta Otolaryngol. 83, 85 (1977)

11. §. Flock, and H.Cheung, J. Cell Biol. 75, 339 (1977)

12. D.J.Derosier, L.G. Tilney and E. Egelman, Nature 287, 291 (1980)

13. M.A.Sleigh, The Biology of Cilia and Flagella, Macmillan, New York, 1962

14. A.Flock, H.C.Cheung, B.Flock and G.Utter, J. Neurocytology 10, 133 (1981)

15. 0 . Lowenstein and J. Wershl1, Nature 184, 1807 (1959)

16. A.Flock, J. Cell Biol. 22, 413 (1964)

17. A. Flock, Cold Spring Harbor Symposia on Quantitative Biology 30,133 (1965)

18. H. Spoendlin, in: The Vestibular System and Its Diseases, University of Pennsylvania Press, Philadelphia, 1966, p. 39

19. H. Engstrym and B. Engstrbm, in: The Vestibular System: Function and Morphology, SpringerVerlag, New York, 1981, p. I

20. H.P.Zenner, Arch. Otorhinolaryngol. 230, 81 (1981) 
21. G.L.Rasmussen, in: Neural Mechanisms of the Auditory and Vestibular Systems, Thomas, Springfield, 1960, p. 105

22. M.D.Ross, in: The Vestibular System: Function and Morphology, Springer-Verlag, New York, 1981 , P. 160

23. H.H.Lindeman, Adv. Otorhinolaryngol. 20, 405 (1973)

24. M.D. Ross and D.Peacor, Ann. Otol. 84,22 (1975)

25. D. Carlstrym, H. Engstrom and S. Hjorth, Laryngoscope 63, 1052 (1953)

26. D.B.Peacor, R.C.Rouse and M.D.Ross, Anat. Rec. 197,375 (1980)

27. H. Nakahara and G. Bevelander, Anat. Rec. 193, 233 (1979)

28. E. Fukuda and I.Yasuda, J. Phys. Soc. Japan 12, 1158 (1957)

29. R.W.Morris and L.R.Kitt Ieman, Science 158,368 (1958)

30. C.A.Smith, O.H.Lowry and M.L.Wu, Laryngoscope 64, 141 (1954)

31. D.Trincker, Pf1Ugers Arch. ges. Phys1ol. 264, 351, 1957

32. G. von Bekesy; Nature 169, 241 (1952)

33. I.J.Rusaell and P.M.Sellick, J. Physiol. (Lond.) 284, 261, 1978

34. V.J.Wilson and G.Melvill Jones, Mammalian Vestibular Physiology, Plenum Press, New York, 1979

35. A.J.Hudspeth, J. Neurosc1. 2, I (1982)

36. A. Flock, 1n: The Vestibular System: Function and Morphology, Springer-Verlag, New York, 1981, p. 275 .

37. H.H.Lindeman, Adv. Anat. Embryol. Cell. Biol. 42, 1 (1969)

38. W.J.Cady, Plezoelectricity, Dover Publications, New York, 1964

39. H. Athenstaedt, Archs. oral Biol. 16, 495 (1971)

40. A.L.Bassett, Calc. Tiss. Res. 1,242 (1968)

41. M.D.Ross, K.G.Pote, K.E.Rarey and L.M.Verma, Ann. N.Y. Acad. Sci. 374, 808 (1981)

42. G.B.Wislocki and A.J.Ladman, J. Anat. 89,3 (1955)

43. M.D.Ross, Anat. Rec. 175,449 (1973)

44. D. E.Green, Ann. N.Y. Acad. Scf. 227, 6 (1974)

45. F. Gutmann and L.E.Lyons, Organic Semiconductors, John Wiley, New York, 1967

46. B. Roseaberg, J. chem. Phys. 36, 816 (1962)

47. J.J.Wolken, Euglena, 2nd ed., Appleton-Century Crofts, New York, 1967

48. S.V.Perry, symp. Soc. exp. Biol. 22, 1 (1968)

49. K. Ludloff, Pfillg. Arch. ges. Physiol. 59, 525 (1895)

50. R. Wichtermann, The Biology of Paramecium, Blakiston, Philadelphia, 1953

51. T.L.Jahn, J. Protozool. 7, 16 (1960)

52. T.L.Jahn, J. Protozool. 8,369 (1961)

53. E.C.Bovee and T.L.Jahn, J. theor. Biol. 35, 259 (1972)

54. M.S.Moeseker and L.G.Tilney, J. Cell Bio1. 67, 725 (1975)

55. M. Schliwa, Cell 25,587 (1981)

56. A. Weeds, Nature $\frac{256}{29}, 811$ (1982)

57. 0. Lowenstein, J. Physiol. 127, 104 (1955)

58. T. Furukawa and Y. Ishii, Jap. J. Physiol. 17, 572 (1967)

59. T. Furukawa and $Y$. Ishii, J. Neurophysiol. 3 30, 1377 (1967)

60. I. Tasaki and C. Fernandez, J. Neurophysiol. 15, 497 (1952)

61. T. Konish1, D.C.Teas and J.S.Wernick, J. Acoust. Soc. Am. 47, 1519 (1970)

62. D.C.Teas, T. Konishi and J.S.Wernick, J. Acoust. Soc. Am. 47, 1527 (1970)

63. J. M. Aran, Brit. J. Lasyngol. Otol. 95, 153, 1981

64. G.C.Offutt, J. Aud. Res. 10,226 (1970)

65. F. Bracchi, T. Gualtierot ti, A. Morabito and E. Rocca, Acta Laryngol. Suppl. 334 (1975)

66. M.D.Ross, K.G.Pote, P.L.Cloke and C.Corson, The Physiologist 23, S-129 (1980)

67. A. Guardabass1, Z. Zellforsch. 51, 278 (1960)

68. T. Imoto and H. Rask-Andersen (in press, 1982)

69. M. J. Buerger, Elementary Crystallography, Wiley, London, 1956

70. H. Athenstaedt, Nature 228, 830 (1970) 